\title{
Modeling Parking Behavior Under Uncertainty: A Static Game Theoretic versus a Sequential Neo-additive Capacity Modeling Approach
}

\author{
Liya Guo • Shan Huang • Jun Zhuang • \\ Adel W. Sadek
}

Published online: 30 December 2012

(C) Springer Science+Business Media New York 2012

\begin{abstract}
This paper proposes two types of parking choice models, a static game theoretic model and a dynamic neo-additive capacity model, to capture the competition among drivers for limited desirable parking spaces. The static game assumes that drives make decisions simultaneously and with perfect knowledge about the characteristics of the parking system and the strategies of their fellow drivers in the system; the model thus captures only the rational aspect of parking choice behavior and pays no attention to modeling individual drivers' psychological characteristics. The dynamic model, on the other hand, considers individual drivers' psychological characteristics under uncertainty (i.e. optimistic and pessimistic attitudes) and thus captures the impacts of the irrational side of parking behavior in addition to the rational aspect. Following the formulation of the two models, they are both used to predict parking behavior as observed on a set of parking lots on the University at Buffalo north campus. Specifically for the dynamic model, the model is first calibrated based on real data collected from video recorded observations for a pair of parking lots, and then used to predict behavior on another pair. Validation results show higher predictive accuracy for the dynamic neo-additive capacity model compared to the static game theoretic model. This in turn suggests that the psychological characteristics of drivers play an important role in the parking lot choice decision process, and points to the potential for parking information systems to eliminate the unnecessary additional traffic generated by the parking search process.
\end{abstract}

Keywords Parking behavior - Game theory · Decision analysis · Uncertainty modeling · Simulation

L. Guo $\cdot$ S. Huang $\cdot$ A. W. Sadek $(\bowtie)$

Department of Civil, Structural, and Environmental Engineering, University at Buffalo, The State University of New York, Buffalo, NY 14260, USA

e-mail: asadek@buffalo.edu

J. Zhuang

Department of Industrial and Systems Engineering, University at Buffalo, The State University of New York, Buffalo, NY 14260, USA 


\section{Introduction}

Parking planning and management is a critical need for modern cities. Since the 1990s, traffic caused by parking search has been recognized as a significant contributor to the urban congestion problem (Polak and Axhausen 1990). In their review, Polak and Axhausen (1990) state that parking behavior results from a complex interaction process between the individual characteristics of drivers (the demand side) and parking opportunities (the supply side). They also conclude that travelers adopt various strategies based on their previous parking search experiences and the performance of the transportation system. Given the complex nature of the parking choice process, drivers' parking behavior is still not well understood, despite several previous studies reported in the literature. Those studies are briefly reviewed below.

Parking search models reported in the literature can be categorized into two groups: (1) models for parking search within one specific parking lot; and (2) models of drivers' choice of a given parking lot from among several alternative lots. With respect to the first category (i.e. search within one lot), one of the earliest models for simulating such behavior is the PARKSIM system developed by Young (1986). One limitation of that model, however, is that all drivers are assumed to adopt the same parking strategy. Cassady and Kobza's (1998) probablistic approach improves on Young's framework by defining two different parking strategies and assuming parking space availability probabilities. More recent simulation-based models (e.g. Young and Weng 2005; Chalamish et al. 2007; Leephakpreeda 2007) simulate the interaction between the transportation system and the drivers who want to minimize their own costs. They do not however include analytical representations of the underlying behavioral processes.

With respect to modeling driver's choice of a given parking lot from among several alternatives, which is the focus of the work described in this paper, Thompson and Richardson (1998) categorized earlier models into utility models (e.g. Van Der Goot 1982; Hunt 1988), optimization-based models (e.g. Goyal and Gomes 1984; Tsukaguchi and Jung 1989), distribution models (e.g. Ellis and Rassam 1970; Florian and Los 1980; Young, et al. 1991) and assignment models (e.g. Nour Eldin et al. 1981; Gur and Beimborn 1984). Thompson and Richardson (1998) also pointed out several behavioral deficiencies with those earlier models including unrealistic assumptions of perfect information, no learning knowledge, and no temporal or dynamic aspects in the choice process. Khattak and Polak (1993) provided a conceptual structure for the parking choice decision making process, which incorporates factors such as parking information, parking facility attributes, individual characteristics, trip characteristics and situational factors. However, they admitted that the factors influencing "imperfection of knowledge" (i.e. uncertainty) were not clearly understood.

Following their review of parking choice modeling literature, Thompson and Richardson's (1998) proceeded to develop their own parking search model, which significantly advanced the state-of-the-art, by adapting the economic search principle of expected gain in utility, to represent the searching patterns of drivers. Their study especially focused on how the costs associated with the attributes of a parking lot (e.g., occupancy, geometric characteristics, payment, waiting time, etc.) combine to constitute an overall measure of utility. Although the model provided a detailed analytical representation, it still lacked consideration of driver's psychological factors and thus was unable to replicate the observed irrational aspect of parking behavior, whose importance 
was emphasized by Khattak and Polak (1993). Specifically, Thompson \& Richardson assumed that the perceived utility of a parking lot was constant for all drivers. In reality, however, drivers with different personalities make quite different decisions when they face the same situation. Therefore, individual characteristics should not be ignored when modeling parking behavior. In addition, Thompson and Richardson's (1998) model was a discrete choice model, and thus ignored the interactions between the outcome of a driver's parking choice and the parking lot availability.

This paper proposes a game theoretic model with both static and dynamic formulations for modeling drivers' parking choice behavior among alternative parking lots. The static game borrows the concept and notations of the perfect game theoretic model developed by Wang and Zhuang (2011). The model is designed to capture only the rational aspect of parking choice behavior and pays no attention to modeling individual drivers' psychological characteristics. Moreover, it is concerned with predicting the percentage of drivers that go to a given parking lot, rather than predicting the individual choice of a certain driver with certain psychological characteristics. The more significant contribution of the paper is in its development of the dynamic model, which includes a custom-defined non-extreme-outcome-additive capacity (neo-additive capacity) formulation (Chateauneuf, et al. 2007). The inclusion of the neo-additive capacity formulation allows the model to capture differences among individual drivers' psychological characteristics (e.g. optimistic and pessimistic attitudes) towards a parking system's availability under uncertainty. Following the formulation of the two models, they are both used, in a case study, to predict parking behavior as observed on a set of parking lots on the University at Buffalo (UB), the State University of New York (SUNY) north campus. For that case study, the dynamic model is first calibrated based on real data collected from video recorded observations for a pair of parking lots, and then used to predict behavior on another pair. A comparison is then made between the predictions of the static and the dynamic neo-additive capacity model to assess the significance of the irrational aspect of parking choice behavior which reflects individual drivers' psychological characteristics.

The rest of this paper is organized as follows. Section 2 formulates the static gametheoretic parking choice model. Section 3 formulates the dynamic model, and explains the fundamental theories underpinning the model's formulation and the different parameters capturing the inherent uncertainty associated with the parking choice process and the drivers' psychological differences or attitudes toward risk (i.e. optimism or pessimism). Section 4 then provides the details of applying the two models to the case study. The validation results of the static and dynamic parking choice models, along with the results of a few sensitivity analyses performed on both models, are then presented in Section 5. The paper concludes by summarizing the major findings of the study and providing suggestions for future research in Section 6.

\section{A static game-theoretic model}

\subsection{Model setup}

While game theory (Von Neumann and Morgenstern 1944) has been applied to various problems in transportation before (e.g., Altman and Wynter 2004; Lin and 
Hsieh 2012; Zhang et al. 2005), to the best of the authors' knowledge, the current work represents the first attempt to formulate game theoretic parking choice models. Specifically, the static game-theoretic parking choice model proposed herein considers a population of strategic drivers who seek to park in their desired lots close to their destination building or activity location. The model assumes that drivers (1) are homogenous and rational, (2) desire to minimize their walking distances from where they park to their destination building or activity location, however, (3) they may not want to carry out multiple searches among parking lots. This means that while parking lots close to the destination buildings are more desirable to drivers, the possibility of not being able to find an empty parking spot there, may deter some drivers from going there in the first place to avoid the risk of wasting time in additional parking search processes. The static model further assumes that drivers have perfect knowledge of the characteristics of the parking system, the time costs, and the strategies of their fellow drivers. Based on this, drivers make decisions on whether to compete for the limited spaces in their desirable parking lots by weighing the expected time costs of both options (i.e., competing vs. non-competing). Those decisions are made independently and simultaneously by all drivers, who as a result would not know whether a parking lot is full or not.

To more clearly explain the game-theoretic parking model developed in this paper, the study focuses on a group of drivers who share one common destination building and make their choices between two parking lots (the desired lot $\mathrm{L}_{1}$ and the union of all other alternative lots $\mathrm{L}_{2}$ ). In other words, $\mathrm{L}_{1}$ is always the parking lot closest to the destination building, while $\mathrm{L}_{2}$ is the union of all the remaining alternative lots which are farther away from the building than $\mathrm{L}_{1}$. It is therefore logical to assume that $\mathrm{L}_{2}$ has a much larger physical capacity, which means that empty parking spaces would practically always be available. It should be noted, however, that the division of the available parking lots into just two sets, the desired set $\mathrm{L}_{1}$ and the alternative set $\mathrm{L}_{2}$, does not really limit the application of the framework to cases with only two choices or two parking lots. For cases with more than two lots, a hierarchical decision-making process could be easily employed, whereby the most desirable parking lot is first picked as L1 and the other parking lots are aggregated as L2. If a driver were to choose to go to $\mathrm{L}_{2}$, then $\mathrm{L}_{2}$ would itself be then split into a desired lot and an alternative set of lots, and a similar decision process would then be repeated.

Based on the afore-mentioned assumptions, the following paragraphs will describe the elements of the model. Table 1 lists the major variables and notations used in the model formulation.

\subsubsection{Parking lot characteristics}

We denote the capacity of parking lot $\mathrm{L}_{1}$ by $\mathrm{C}_{1}$, and we assume that, generally speaking, the capacity of parking lot $L_{2}$ (i.e., $C_{2}$ ) is sufficiently larger than $C_{1}$ (i.e., $C_{2}>C_{1}$ ) because parking lot $\mathrm{L}_{2}$ represents the union of all alternate lots other than $\mathrm{L}_{1}$. As previously mentioned, in the static model formulation, drivers are assumed to know the capacity of the two parking lots, along with the probability of being able to find an available spot in their desired parking lot. Naturally, they do not know the actual occupancy of a given lot (and hence they do not know whether a lot is full or not), 
Table 1 Major notations used in the static model formulation

\begin{tabular}{ll}
\hline Notation & Explanation \\
\hline$\Theta$ & Index of parking lot $(\theta \in\{1,2\})$ \\
$\lambda_{\theta}$ & Number of vehicles headed to lot $\theta$ during the analysis period, D (vehicles) \\
$\mu_{\theta}$ & Number of vehicles departing from parking lot $\theta$ during the analysis time period, \\
& D (vehicles) \\
$C_{\theta}$ & Capacity of parking lot $\theta$ (vehicles) \\
$T_{\theta}(\cdot)$ & Expected total time cost associated with choosing parking lot $\theta$ first \\
$\Lambda$ & Number of vehicles arriving at all lots (total parking demand) during time period, \\
& D (vehicles) \\
$p_{i}$ & Parking choice of driver i \\
$\widehat{p}_{i}$ & Best parking strategy for driver i \\
$X_{\theta} \in[0,1]$ & Percentage of drivers who decide to search lot $\theta$ \\
$P\left(X_{1}\right) \in[0,1]$ & Probability of failing to park in $\mathrm{L}_{1}$, given that $\mathrm{X}_{1} \%$ of the total demand head to \\
& parking $\theta_{1}$
\end{tabular}

since all drivers make their decisions simultaneously. The knowledge ambiguity associated with this, and how that ambiguity interacts with the psychological characteristics of individual drivers, are only modeled in the dynamic model formulation described in Section 3 .

\subsubsection{Drivers' characteristics}

We use $\Lambda$ to refer to the total demand for parking (i.e., the total number of vehicles arriving at all parking lots within the parking system during the analysis period, D) and we naturally assume that that demand may vary throughout different time periods of the day. We then define a variable $p_{i} \in[0,1]$ to capture the decision options for each driver $i$. In other words, if $p_{i}=1$, the driver searches $\mathrm{L}_{1}$ first; and if $p_{i}=0$, she/he searches $\mathrm{L}_{2}$ first. With this, we have $X_{1}=\frac{1}{\Lambda} \sum_{i} p_{i}$ representing the percentage of all drivers who choose to search $\mathrm{L}_{1}$ and $X_{2}=1-X_{1}$ representing the percentage of all drivers who go directly to $\mathrm{L}_{2}$. The number of vehicles going to $\mathrm{L}_{1}$ and $\mathrm{L}_{2}$ can then be calculated as $\lambda_{1}=X_{1} \Lambda$ and $\lambda_{2}=$ $X_{2} \Lambda$ respectively. Finally, we define $\mu_{1}$ and $\mu_{2}$ to represent the numbers of vehicle leaving from $\mathrm{L}_{1}$ and $\mathrm{L}_{2}$ during the analysis period. In this paper, we focus on periods of peak parking demand where the arrival rate to the parking system is significantly larger than the departure (i.e. $\lambda_{1}>>\mu_{1}$ and $\lambda_{2}>>\mu_{2}$ ).

\subsubsection{Time costs}

A driver who searches $L_{1}$ will incur time cost $t_{1}$ if she/he successfully parks in $L_{1}$, however, if the driver fails to find an available spot because $\mathrm{L}_{1}$ is full, the driver would then need to travel back to $\mathrm{L}_{2}$, and as a result would incur the extra time cost $t_{3}$ (which includes the search time within $\mathrm{L}_{1}$ and the travel time from $\mathrm{L}_{1}$ to $\mathrm{L}_{2}$ ). In contrast, a driver who goes straight away to $\mathrm{L}_{2}$ will always find an empty space there, and thus will incur a time cost $t_{2}$, which includes a longer walking time to the 
destination building compared to $t_{1}$. To make the analysis tractable, the magnitudes of time costs above would have to satisfy the following inequality: $t_{1}<t_{2}<\left(t_{2}+t_{3}\right)$.

Given the above, the expected time cost associated with choosing $\mathrm{L}_{1}, T_{1}\left(X_{1}\right)$, can be calculated according to Eq. (1).

$$
T_{1}\left(X_{1}\right)=\left[1-P\left(X_{1}\right)\right] \cdot t_{1}+P\left(X_{1}\right) \cdot\left(t_{2}+t_{3}\right)=t_{1}+P\left(X_{1}\right) \cdot\left(t_{2}+t_{3}-t_{1}\right)
$$

where, $P\left(X_{1}\right) \in[0,1]$ denotes the probability of failing to find a space in $\mathrm{L}_{1}$ when the percentage of drivers searching $\mathrm{L}_{1}$ is $X_{1}$ of the total demand, $\Lambda$. As can be seen, the first term of Eq. (1) captures the case of those drivers who manage to find a spot in $\mathrm{L}_{1}$, whereas the second term of the equation is for those drivers who fail to find a spot in $\mathrm{L}_{1}$ and therefore have to travel back to $\mathrm{L}_{2}$ to park there. Obviously, $P\left(X_{1}\right) \in[0,1]$ only when the number of drivers searching $L_{1}$ is greater than the sum of the capacity of $L_{1}$ and the additional spaces vacated by the vehicles departing from $\mathrm{L}_{1}$; this is expressed mathematically as, $\lambda_{1}=X_{1} \Lambda>\left(\mu_{1}+C_{1}\right)$. In that case, some drivers choosing $\mathrm{L}_{1}$ are unable to park in $\mathrm{L}_{1}$ because it is full. On the other hand, when $X_{1} \Lambda \leq\left(\mu_{1}+C_{1}\right)$, all drivers choosing $\mathrm{L}_{1}$ would be able to park there. $P\left(X_{1}\right)$ can thus be calculated as in Eq. (2).

$$
P\left(X_{1}\right)= \begin{cases}1-\frac{\mu_{1}+C_{1}}{\Lambda X_{1}} & \frac{\mu_{1}+C_{1}}{\Lambda}<X_{1} \leq 1 \\ 0 & 0 \leq X_{1} \leq \frac{\mu_{1}+C_{1}}{\Lambda}\end{cases}
$$

For the drivers who go directly to $\mathrm{L}_{2}$, their expected time cost $T_{2}\left(X_{2}\right)$ is a constant, as shown in Eq. (3).

$$
T_{2}\left(X_{2}\right)=t_{2}
$$

\subsection{Definition of equilibrium or convergence}

Based on the assumption that each driver hopes to minimize his or her expected time cost, the best strategy $\widehat{p}_{i}$ for driver $i$ is given as in Eq. (4):

$$
\widehat{p}_{i}= \begin{cases}1 & \text { if } \mathrm{T}_{1}\left(\mathrm{X}_{1}\right)<\mathrm{T}_{2}\left(\mathrm{X}_{2}\right) \\ 0 & \text { if } \mathrm{T}_{1}\left(\mathrm{X}_{1}\right)>\mathrm{T}_{2}\left(\mathrm{X}_{2}\right) \\ \in(0,1) & \text { if } \mathrm{T}_{1}\left(\mathrm{X}_{1}\right)=\mathrm{T}_{2}\left(\mathrm{X}_{2}\right)\end{cases}
$$

Therefore, only if $T_{1}\left(X_{1}\right)=T_{2}\left(X_{2}\right)$, would going to $\mathrm{L}_{1}$ and $\mathrm{L}_{2}$ be both regarded as best strategies for drivers, and hence a state of equilibrium may be assumed to exist with no driver having a better strategy to lower parking cost by switching parking choices. Based on Eqs. (1), (2), (3), and (4), therefore, the percentage of drivers choosing to go to lot $\mathrm{L}_{1}$ first, at equilibrium, may be calculated by setting Eq. (1) equal to Eq. (3). This results in:

$$
X_{1}^{*}=\frac{\left(t_{2}+t_{3}-t_{1}\right)\left(\mu_{1}+C_{1}\right)}{t_{3} \Lambda}
$$

Note that for Eq. (5), and given that $X_{1}$ has to be equal to or less than 1.0, the condition that $\Lambda \geq \frac{\left(t_{2}+t_{3}-t_{1}\right)\left(\mu_{1}+C_{1}\right)}{t_{3}}$ has to be satisfied, otherwise, $X_{1}{ }^{*}$ would have to equal to 1.0. With this, the values of $X_{1}{ }^{*}$ at equilibrium, and the conditions for that equilibrium to be valid can be summarized as shown in Table 2 . 


\section{A dynamic neo-additive capacity model}

The previous section focused on a static parking game formulation, based on the assumptions that all drivers are identically rational and make decisions simultaneously. Building on this, the current section formulates a dynamic model formulation which relaxes the assumptions above and views the parking game as a sequential process under the uncertainty regarding a parking lot's availability. The aim of the dynamic model is to capture the heterogeneity among drivers in terms of their parking behaviors and attitudes toward risk. Specifically, as opposed to the static model which captures the "rational" aspect of parking choice decision-making, the dynamic model reflects the "irrational" or subjective attitudes towards uncertainty (ambiguity), such as pessimism and optimism.

\subsection{Model setup}

\subsubsection{The neo-additive capacity concept}

Different types of people show different attitudes when facing uncertainty. An optimistic person overestimates the likelihood of good outcomes while a pessimistic person exaggerates the likelihood of bad outcomes. To account for this, Chateauneuf et al. (2007) improved the rank-dependent Choquet-expected utility (CEU) model (Schmeidler 1989) by introducing the concept of neo-additive capacity, which considers both the rational attitude as well as the irrational attitude (pessimism and optimism) towards uncertainty (Wakker 2001). The dynamic parking choice model proposed in this study makes use of Chateauneuf's formulation.

As shown in Fig. 1, an individual's decision-making process can be viewed as containing aspects of both rational and irrational behavior. If the information is clear and precise, the decision maker can make a completely rational decision. Otherwise, the irrational aspect plays its role. A constant $\delta$ named "ambiguity factor" is introduced to represent the degree of unknown information for a person's perception. $\delta$ is defined to be in the range $[0,1]$, where $\delta=0$ means perfect information is available to the decision maker, and hence he/she should adopt strategies purely based on expected utility (EU) theory. When $\delta$ increases, the portion of ambiguous knowledge also increases. $\delta=1$ refers to the case of complete ignorance about the situation.

When dealing with ambiguity, people often make decisions according to their personalities. The irrational component of decision-making thus includes two psychological aspects: optimism (considering the best result) and pessimism (considering

Table 2 The equilibrium of the static game

\begin{tabular}{ll}
\hline Conditions & $X_{1}^{\mathrm{a}}$ \\
\hline$\Lambda<\left(\mu_{1}+C_{1}\right) \& t_{1}<t_{2}<\left(t_{2}+t_{3}\right)$ & 1 \\
$\left(\mu_{1}+C_{1}\right) \leq \Lambda<\frac{\left(t_{2}+t_{3}-t_{1}\right)\left(\mu_{1}+C_{1}\right)}{t_{3}} \& t_{1}<t_{2}<\left(t_{2}+t_{3}\right)$ & $1^{\mathrm{a}}$ \\
$\Lambda \geq \frac{\left(t_{2}+t_{3}-t_{1}\right)\left(\mu_{1}+C_{1}\right)}{t_{3}} \& t_{1}<t_{2}<\left(t_{2}+t_{3}\right)$ & $\frac{\left(t_{2}+t_{3}-t_{1}\right)\left(\mu_{1}+C_{1}\right)}{t_{3} \Lambda}$
\end{tabular}

\footnotetext{
${ }^{\text {a }}$ Note that this condition represents the case where the probability of failing to find an available spot in $\mathrm{L}_{1}$ is so small that it is still more advantageous, from an expected total time cost standpoint, to go to $\mathrm{L}_{1}$ first
} 
Fig. 1 Decomposition of individual characteristics

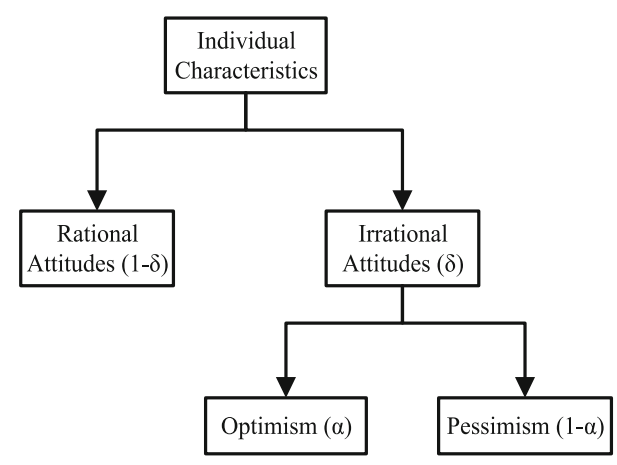

the worst result). A person has his/her own way of balancing these two aspects, by giving them different weights. We use $\alpha$ to denote the degree of optimism, as is also shown in Fig. 1. For a pure optimist, $\alpha$ is closer to 1 ; otherwise $\alpha$ is closer to 0 . Pessimism can therefore be defined as $1-\alpha$.

Considering both the degree of ambiguity and the degree of optimism, the neoadditive capacity, $v$, is then formulated as shown in Eq. (6).

$$
\begin{aligned}
v= & \delta \cdot\left(\alpha \cdot \max \left\{u\left(x_{1}\right), \ldots, u\left(x_{n}\right)\right\}+(1-\alpha) \cdot \min \left\{u\left(x_{1}\right), \ldots, u\left(x_{n}\right)\right\}\right) \\
& +(1-\delta) \cdot \sum_{m=1}^{n} \pi_{m} u\left(x_{m}\right)
\end{aligned}
$$

Where, $u($.$) is the expected utility function, and \pi_{m}$ are decision weights satisfying the condition that $\sum_{m=1}^{n} \pi_{m}=1$. Details regarding this formulation can be found in Chateauneuf, et al. (2007), and in Sarin and Wakker (1998).

\subsubsection{Neo-additive capacity formulation of the parking choice game}

Based on Eq. (6), the neo-additive capacities for the dynamic parking game can be defined, as shown in Eqs. (7) and (8). Note that besides the already mentioned time cost notations, all notations used in the dynamic model are summarized in Table 3.

$$
\begin{gathered}
v_{i 1}=\delta \cdot\left[\alpha_{i} \cdot \min \left\{t_{1},\left(t_{2}+t_{3}\right)\right\}+\left(1-\alpha_{i}\right) \cdot \max \left\{t_{1},\left(t_{2}+t_{3}\right)\right\}\right]+(1-\delta) \\
\cdot\left[\left(1-\pi_{i}\right) t_{1}+\pi_{i} \cdot\left(t_{2}+t_{3}\right)\right]=\delta \cdot\left[\alpha_{i} \cdot t_{1}+\left(1-\alpha_{i}\right) \cdot\left(t_{2}+t_{3}\right)\right] \\
+(1-\delta) \cdot\left[t_{1}+\pi_{i} \cdot\left(t_{2}+t_{3}-t_{1}\right)\right] \\
v_{i 2}=t_{2}
\end{gathered}
$$

The drivers will therefore make their decisions by comparing the capacity values, $v_{i 1}$ and $v_{i 2}$. Note that the capacity here is defined based on disutility, and thus each driver will choose the parking lot associated with the smaller capacity value, which is in essence the smaller time cost. For example, when $v_{i 1}<v_{i 2}$, the driver $i$ chooses to go to $\mathrm{L}_{1}$, otherwise he/she goes to $\mathrm{L}_{2}$. Also note that the formulation of Chateauneuf et 
Table 3 Major notations for the dynamic model

\begin{tabular}{ll}
\hline Notation & Explanation \\
\hline$\theta \in\{1,2\}$ & Index of parking lot \\
$\mathrm{i} \in\{1,2 . ., \mathrm{n}\}$ & Index of driver \\
$\delta \in[0,1]$ & Ambiguity factor \\
$\alpha_{i} \in[0,1]$ & Index of optimism of driver i \\
$O_{o} \in[0,1]$ & Initial occupancy of $\mathrm{L}_{1}$ \\
$O_{i} \in[0,1]$ & Occupancy of $\mathrm{L}_{1}$ after driver $i$ makes his/her parking lot choice \\
$\pi_{i} \in[0,1]$ & Driver i's perceived probability of $\mathrm{L}_{1}$ being already full \\
$\nu_{i \theta}$ & Driver i's neo-additive capacity of searching lot $\theta$ \\
$\gamma$ & Factor determines how pronounced the inverse-S shape curve is \\
$p_{i}\left(\alpha_{i}, O_{i-1}\right) \in\{0,1\}$ & Driver i's choice \\
$g_{i}\left(\alpha_{i}, O_{i-1}\right) \in\{0,1\}$ & Outcome of driver i's choice in terms of the incremental increase in $O_{i}$ \\
\hline
\end{tabular}

al. (2007), previously shown in Eq. (6), is based on utiltiy, and hence an individual chooses the option with larger capacity value. This is the reason behind the switching of the min and max operators between Eqs. (6) and (7).

\subsubsection{Uncertainty, optimism, and pessimism parameters}

In our dynamic parking model, we assume $\delta$ to be an unknown constant, and $\alpha$ to be normally distributed among drivers with a mean $\mu$ and a variance $\sigma^{2}$. In other words, for an individual driver $\mathrm{i}$, his/her $\alpha_{i} \sim N\left(\mu, \sigma^{2}\right)$. However, given that $\alpha_{i}$ has to lie between 0 and 1 , the range of the normal distribution is set to that range and adaptively limited to within $[\mu-3 \sigma, \mu+3 \sigma]$, which covers $99.7 \%$ of the values. It should be noted that, if desired, the proposed modeling framework can be easily extended to the case where the ambiguity factor, $\delta$, also varies among drivers. This variation could be used to reflect, for example, different levels of access to information about parking availability through different levels of prior experience with the parking system and the associated learning process. In such cases, an appropriate probability distribution would have to be assumed for $\delta$.

\subsubsection{The probability of the first-choice parking lot being full}

When a certain driver is making a choice between $\mathrm{L}_{1}$ and $\mathrm{L}_{2}$, the probability of $\mathrm{L}_{1}$ being already full should be expected to influence the driver's decision. It can therefore be safely assumed that a driver's perceived probability of $\mathrm{L}_{1}$ being full is a key factor in choosing whether to go to parking lot $\mathrm{L}_{1}$ or $\mathrm{L}_{2}$. In our model formulation, we use that perceived probability as the decision weight (i.e. the $\pi_{i}$ ) of the neo-additive capacity formulation of Eq. (7). The probability of a parking lot being full is naturally related to the current occupancy of the lot (i.e. the total number of vehicles currently parked divided by the total capacity of the lot), which we denote by $O$. However, because drivers, in the absence of parking guidance system, typically 
do not have complete information about the $\mathrm{L}_{1}$ 's occupancy, their perceived probability of a parking lot being full must be assumed to be different from that in reality.

A formulation is therefore needed to calculate the perceived probability of $\mathrm{L}_{1}$ being full $(\pi)$ from the occupancy of the parking lot $(O)$; obviously, $\pi$ and $O$ are both required to be within the range of $[0,1]$. Moreover, it can be assumed, that for a given driver, the concern about finding an available parking space becomes an issue when the occupancy of the parking lot is approaching its maximum capacity. Based on the aforementioned assumption, $\pi$ should grow slowly until $O$ is getting close to 1 . We therefore calculate $\pi$ using an inverse-S-shaped Quiggin function (Tversky and Kahneman 1992), as shown in Eq. (9).

$$
\pi(O)=\frac{O^{\gamma}}{\left[O^{\gamma}+(1-O)^{\gamma}\right]^{1 / \gamma}}
$$

where, $\gamma$ is a factor which determines how pronounced the inverse-S shape curve is.

Based on empirical information, De Palma et al. (2008) stated that the recommended range for the factor, $\gamma$, is between 0.279 and 1 . In this paper, we therefore assume a value for $\gamma$ close to its lower bound; specifically, we assume $\gamma$ to be equal to 0.3. The reason for doing this is best explained by looking at Fig. 2, which plots the curves of $\pi$ versus $O$ for different $\gamma$ values, compared to a 45-degree straight line (which corresponds to a value of $\gamma$ equal to 1.0). As can be seen, the inverse-S shape generated by $\gamma=0.3$ captures the phenomenon we are interested in modeling. With this curve, our model can reflect the fact that drivers start getting concerned about $\mathrm{L}_{1}$ being full (i.e. $\pi$ starts increasing significantly), only after $\mathrm{O}$ reaches a value above 0.9 . For lower values of $O, \pi$ maintains quite small values.

\subsubsection{A sequential gaming process}

We now define two variables $p_{i}\left(\alpha_{i}, O_{i-1}\right)$ and $g_{i}\left(\alpha_{i}, O_{i-1}\right)$, as shown in Eqs. (10) and (11) respectively. The variable $p_{i}\left(\alpha_{i}, O_{i-1}\right)$ captures the decision made by a given

Fig. 2 Perceived probability of L1 being full versus L1's occupancy

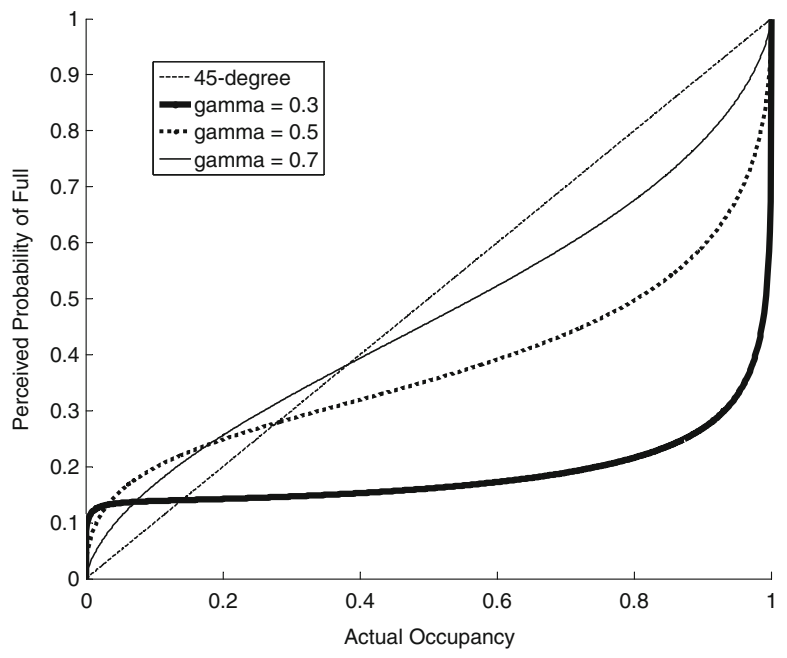


driver $i$ with $\alpha_{i}$ degree of optimism. Similar to the static model, $p_{i}$ is set to 1.0 if the driver chooses to go to $\mathrm{L}_{1}$, and it is equal to 0 , if he/she decides to go to $\mathrm{L}_{2}$. The variable $g_{i}\left(\alpha_{i}, O_{i-1}\right)$ on the other hand captures the outcome in terms of the incremental increase in the number of cars parked in parking lot $\mathrm{L}_{1}$. Specifically, $g_{i}\left(\alpha_{i}, O_{i-1}\right)$ is equal to 1.0 if the driver goes to parking lot $\mathrm{L}_{1}$, and manages to park there, otherwise 0 . Considering a single driver $i$ in this game, and given his/her $\alpha_{i}$, and the occupancy of parking lot $\mathrm{L}_{1}$ after the previous driver $(i-1)$ had made his/her decision, $O_{i-1}$, the values of the two variables can be calculated by comparing the corresponding neoadditive capacity values, as follows.

$$
\begin{gathered}
p_{i}\left(\alpha_{i}, O_{i-1}\right)= \begin{cases}1, & v_{i 1}<v_{i 2} \\
0, & v_{i 1} \geq v_{i 2}\end{cases} \\
g_{i}\left(\alpha_{i}, O_{i-1}\right)=\left\{\begin{array}{cc}
1, & \text { if } O_{\mathrm{i}-1}<1 \& v_{i 1}<v_{i 2} ; \\
0, & \text { if } O_{\mathrm{i}-1}=1 ;
\end{array}\right.
\end{gathered}
$$

Now consider a permutation $\left(\alpha_{1}, \alpha_{2} \ldots, \alpha_{n}\right)$ which captures the psychological attitudes of a total of $n$ drivers in terms of their optimistic index $\alpha$, and an initial occupancy of $\mathrm{L}_{1}$ denoted by $O_{0}\left(O_{0}<1\right)$. Once the parking game starts, players make their choices one by one. If driver i chooses $\mathrm{L}_{1}$ and finds a space therein successfully, then the $O_{i}$ will be increased by one unit from $O_{i-1}$; otherwise $O_{i}=O_{i-1}$. The increment in $\mathrm{L}_{1}$ 's occupancy affects the perceived probability of the parking lot $\mathrm{L}_{1}$ being full $\pi_{i}$ ${ }_{+1}\left(O_{i}\right)$ for the next driver $\mathrm{i}+1$, and thereby affects his/her disutility and the neoadditive capacity values, and finally affects his/her parking lot choice. This means that the moves of subsequent players are affected by the moves of their antecedents. This is demonstrated by Eqs. (12) through (14).

$$
\begin{gathered}
O_{1}=O_{0}+g_{1}\left(\alpha_{1}, p_{0}\right) / C_{1}, \\
O_{2}=O_{1}+g_{2}\left(\alpha_{2}, O_{1}\right) / C_{1}, \\
O_{i}=O_{i-1}+g_{i}\left(\alpha_{i}, O_{i-1}\right) / C_{1},
\end{gathered}
$$

\subsection{Simulation}

The dynamic model formulated above is designed to be used within a simulation environment to simulate drivers' parking lot choices and the resulting search process. The simulation process is described by the pseudo code shown in Fig. 3.

As can be seen from the pseudo-code, there are three unknown parameters: (1) the ambiguity factor, $\delta$; (2) the mean $\mu$; and (3) the variance $\sigma^{2}$ of the distribution of the driver's index of optimism $\alpha$. Those parameters can be calibrated first based on observed data before using the model to predict drivers' behavior for other scenarios (the calibration process is described in the next subsection). It should also be noted that, to make the game more 
Generate the a attribute for each driver using $\mu$ and $\sigma 2$;

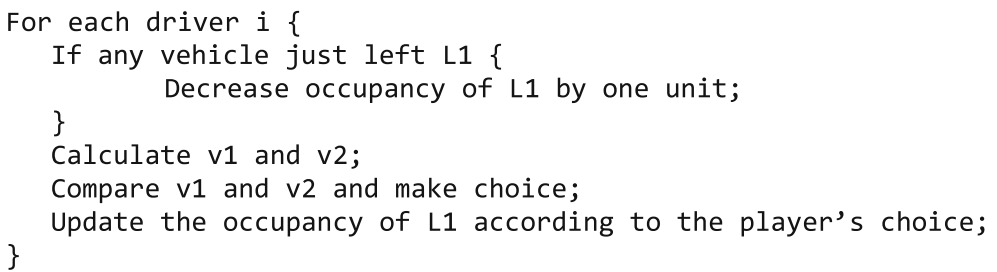

Fig. 3 Pseudo code: simulating the dynamic game

realistic, we also simulate the effect of vehicles departing from parking lot $\mathrm{L}_{1}$ and assume that the departure rate follows a uniform distribution.

\subsection{Model calibration with genetic algorithms}

In this study, a Genetic Algorithm (GA) was developed to calibrate the dynamic model parameters, although other search or optimization methods could of course have been used. The idea was to first calibrate the parameters based on observed drivers' parking lot choice at a set of two parking lots, before using the model to predict drivers' behavior at other lots. As is well known, GAs are stochastic algorithms whose search methods are based on the concept of survival of the fittest, and which have been widely utilized to solve hard optimization problems in transportation and elsewhere (e.g., Michalewicz 1994; Shepherd and Sumalee 2004; Neema et al. 2011). In a typical GA, each possible solution is coded using a data structure, and is called an individual. Many individuals comprise a population. The "fitness" of each individual is then evaluated by a fitness function which gives an index indicating how good the individual is. Using a selection mechanism (to select individuals according to their fitness), the whole population is then evolved from one generation to the next. In this process, each individual is subject to possible alterations by means of genetic operators to include migration (an individual joins the next population without being modified), mutation (an individual joins the next population with a slight modification), and crossover (two individuals creates one offspring which joins the next population). The overall fitness and best fitness of the population are expected to improve over generations, and after several generations, the algorithm is expected to converge and reach a near optimal solution.

Developing a GA to solve a given problem requires: (1) a representation scheme to represent the individuals or the candidate solutions; (2) a fitness function to evaluate the individuals; (3) a procedure to generate the initial population; (4) a procedure for selecting the more fit individuals from a given generation; and (5) mutation and crossover operator. In addition, one needs to specify the GA control parameters, which include the population size (i.e. the number of individuals per generation); (2) the number of GA generations (i.e. iterations of the algorithm); and (3) the probabilities of applying the mutation and crossover operators. A brief description of the aforementioned GA components is given in Table 4. With respect to the GA control parameters, a population size equal to 100 was used, and the GA was run for 20 generations. The probabilities of applying the migration, mutation and crossover operators were $10 \%, 80 \%$, and $10 \%$ respectively. 
Table 4 GA settings

\begin{tabular}{|c|c|c|}
\hline GA Component & Purpose & Definition \\
\hline $\begin{array}{l}\text { Representation } \\
\text { Scheme }\end{array}$ & $\begin{array}{l}\text { To represent each } \\
\text { individual using a } \\
\text { memory structure. }\end{array}$ & $\begin{array}{l}\text { Each individual is represented by a } 3 \text {-element, real- } \\
\text { value coded vector which corresponds to the three } \\
\text { unknown parameters to be calibrated }\left(\delta, \mu, \sigma^{2}\right) \text {. }\end{array}$ \\
\hline Fitness Function & $\begin{array}{l}\text { To calculate the fitness } \\
\text { of each individual. }\end{array}$ & $\begin{array}{l}\text { After simulating the sequential game and simulating } \\
\text { drivers' choices one by one, the fitness of each GA } \\
\text { individual is calculated by averaging the following } \\
\text { two Absolute Percent Errors (APE): (1) the APE } \\
\text { between the game simulated and field observed } \\
\text { numbers of players going to } \mathrm{L}_{1} \text { before it is full; and (2) } \\
\text { the APE of players going to } \mathrm{L}_{1} \text { after it is full. }\end{array}$ \\
\hline Selection Scheme & $\begin{array}{l}\text { To select individuals } \\
\text { according to their } \\
\text { fitness. }\end{array}$ & $\begin{array}{l}\text { A roulette wheel selection procedure is adopted (see } \\
\text { Michalewicz } 1994 \text { for more details). }\end{array}$ \\
\hline $\begin{array}{l}\text { Initial Population } \\
\text { Creation Function }\end{array}$ & $\begin{array}{l}\text { To create the first } \\
\text { generation of } \\
\text { population. }\end{array}$ & $\begin{array}{l}\text { The initial values } \delta \text { and } \mu \text { are generated from a uniform } \\
\text { distribution between } 0 \text { and } 1 \text {. In order to limit the } \\
\text { distribution }[\mu-3 \sigma, \mu+3 \sigma] \text { so as not to go outside the } \\
\text { allowable range of }[0,1] \text {, the } \sigma^{2} \text { value would have to } \\
\text { depend on the value of } \mu \text {. Given this, after a value for } \\
\mu \text { is selected for a given individual, the algorithm first } \\
\text { calculates the minimum distance, d, from } \mu \text { to the } \\
\text { lower (i.e. the } 0 \text { ) or the upper (i.e. the } 1.0 \text { ) bound } \\
\text { allowed for the optimism parameter, } \alpha \text {. In other } \\
\text { words, the distance d is calculated as equal to min }\{\mu \text {, } \\
1-\mu\} . \sigma^{2} \text { is then generated using a uniform distribution } \\
\text { between } 0 \text { and } d^{2} / 9 \text {, so as to ensure that }[\mu-3 \sigma, \mu+3 \sigma] \\
\text { will be within the allowable range of }[0,1] \text {. }\end{array}$ \\
\hline Mutation Function & $\begin{array}{l}\text { To slightly modify } \\
\text { an individual. }\end{array}$ & $\begin{array}{l}\text { The mutation operator works by simply applying a } \\
\text { factor of either } 95 \% \text { or } 105 \% \text { to either } \delta, \mu \text { or } \sigma^{2} \text {. }\end{array}$ \\
\hline Crossover Function & $\begin{array}{l}\text { To create a new } \\
\text { individual based } \\
\text { on two existing } \\
\text { individuals. }\end{array}$ & $\begin{array}{l}\text { The crossover operators works by calculating the } \\
\text { straight average of the elements of two individuals. }\end{array}$ \\
\hline
\end{tabular}

\section{Case study}

\subsection{Parking lots' configuration and characteristics}

As mentioned in Section 1, the developed static and dynamic parking models were applied to a real world case study involving modeling parking behavior on a subset of the parking lots on UB north campus. Specifically, the case involved two datasets of parking choice observations on UB north campus as shown in Fig. 4. As can be seen, each dataset consisted of two lots $\left(\mathrm{L}_{1}\right.$ and $\left.\mathrm{L}_{2}\right)$ connected via one major road. For dataset 1 , the Ketter lot is closer to the campus building compared to the Jarvis A lot, therefore the Ketter lot is used to represent the first choice lot $\mathrm{L}_{1}$ and the Jarvis A lot is regarded as parking lot $\mathrm{L}_{2}$. Similarly, for the second dataset, the Governors B Lot is $\mathrm{L}_{1}$, while the Governors $\mathrm{D}$ lot is $\mathrm{L}_{2}$. It should be noted that, for dataset 2, only the part of the parking lot inside the highlighted box was considered as $\mathrm{L}_{1}$, since the other part, outside that box, is exclusively for campus visitors and is separated from $L_{1}$ via a parking gate. 


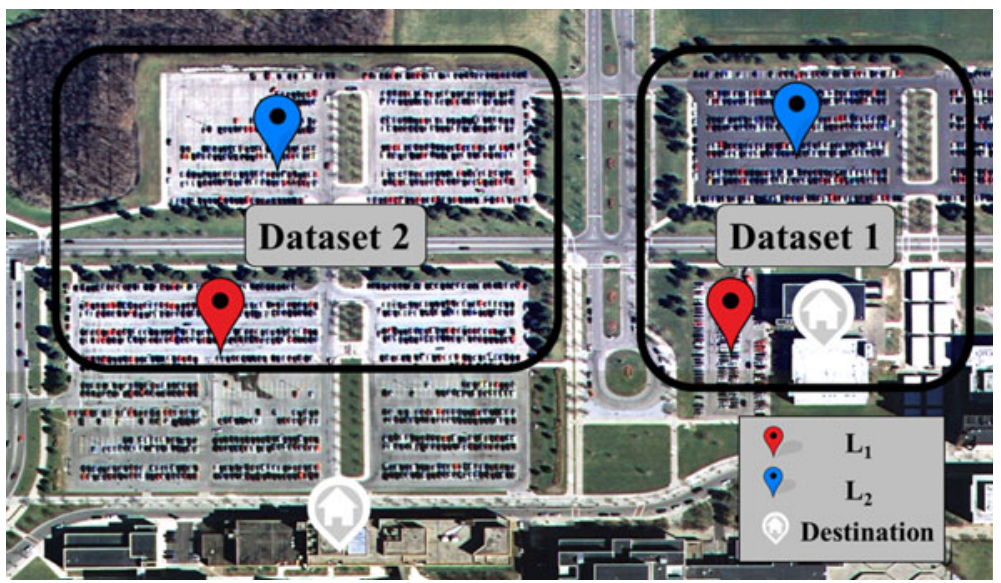

Fig. 4 Location of the two sets of parking lots

Although the parking lot sizes vary between the two datasets, the two parking lot pairs have other similar characteristics and serve the same university population (i.e. faculty, staff and students). The capacity and the initial occupancy of each lot (i.e. occupancy when the parking choice observations started) are shown in the upper half of Table 5 . The table also lists the values for the time costs, $t_{1}, t_{2}$, and $t_{3}$ as observed in the field. The walking time was estimated by having volunteers walk at regular speeds from the geographic center of each parking lot to the destination building, and recording the time taken using a stopwatch. The search time and the time needed to drive from one lot to another were based on the video recordings of parking behavior as described in Section 4.2.

Table 5 Data summary for case study

\section{Dataset 1}

$\mathrm{L}_{1} \quad$ Ketter

$\mathrm{L}_{2} \quad$ Jarvis $\mathrm{A}$

$\mathrm{t}_{1} \quad 36 \mathrm{~s}$

$\mathrm{t}_{2} \quad 110 \mathrm{~s}$

$\mathrm{t}_{3} \quad 58 \mathrm{~s}$

$\mathrm{C}_{1} \quad 113$

O 68

$\begin{array}{cccccc} & 7: 00- & 7: 00- & 7: 00- & 7: 00- & 7: 00- \\ & 7: 30 & 8: 00 & 8: 30 & 9: 00 & 9: 30 \\ \mathrm{~N}_{1} & 22 & 45 & 45 & 45 & 45 \\ \mathrm{~N}_{2} & 24 & 54 & 54 & 54 & 54 \\ \mathrm{~N}_{3} & 0 & 29 & 49 & 78 & 106 \\ \mathrm{~N}_{4} & 0 & 98 & 260 & 424 & 492 \\ \mu_{1} & 1 & 3 & 9 & 16 & 25\end{array}$

Dataset 2

Governors D

Governors B

$75 \mathrm{~s}$

$261 \mathrm{~s}$

$98 \mathrm{~s}$

526

91

$\begin{array}{cccc}9: 00- & 9: 00- & 9: 00- & 9: 00- \\ 9: 15 & 9: 30 & 9: 45 & 10: 00 \\ 120 & 173 & 242 & 286 \\ 2 & 5 & 9 & 18 \\ 0 & 0 & 0 & 0 \\ 0 & 0 & 0 & 0 \\ 3 & 9 & 13 & 16\end{array}$


For the dynamic model, the GA-based calibration uses dataset 1 to determine the three unknown parameters (i.e. $\delta, \mu$, and $\sigma^{2}$ ) that allow the model predictions to best fit the observed parking behaviors. The ability of the calibrated model to replicate drivers' parking choices is then assessed by comparing the model's predictions against field observations not only for the first dataset, but also for the second. This allows the study to assess whether the parameters calibrated for one site are transferrable to other sites.

\subsection{Parking choice data collection}

In order to calibrate and validate the models, video recordings were made of drivers' parking choice behavior at the two sets of parking lots, on a typical Thursday while the university was in session. The data recorded were then manually reduced to extract detailed parking choice and behavior records for each driver entering the parking system. For dataset 1, parking lot choice behavior was observed for a two and a half hours time period, whereas for dataset 2, observations lasted for a full hour. The observed driver choice records were then sliced into 30-min segments for dataset 1 and 15-min segments for dataset 2 . The motivation behind slicing the full observation period into shorter time slices was to increase the number of data points used in model validation and comparisons.

Specifically, for each time slice, the observed data were summarized using the following four key variables: (1) the total number of vehicle entering $\mathrm{L}_{1}$ while there are still parking spaces available in $\mathrm{L}_{1}$, denoted by $\mathrm{N}_{1}$; (2) the total number of vehicle going directly to $\mathrm{L}_{2}$ while there are still parking spaces available in $\mathrm{L}_{1}$, denoted by $\mathrm{N}_{2}$; (3) the total number of vehicle entering $\mathrm{L}_{1}$ when it is full, denoted by $\mathrm{N}_{3}-$ these vehicles would thus fail to find a spot in $\mathrm{L}_{1}$ and would have to switch to $\mathrm{L}_{2}$; and (4) the total number of vehicle going to $\mathrm{L}_{2}$ when $\mathrm{L}_{1}$ is full, denoted by $\mathrm{N}_{4}$. The extracted data are summarized also in Table 5 (the lower half), which also records the numbers of vehicles departing from lot $\mathrm{L}_{1}, \mu_{1}$. As can be seen, the values for $\mu_{1}$ are very small compared to the arrival rates, because observations were taken during the morning peak, when faculty, staff and students are heading toward the university.

\section{Results and discussion}

\subsection{Calibration results}

Using dataset 1 for the time period 7:00-9:30, the three parameters of the dynamic parking choice model were first calculated using GA. As previously described, the GA provides approximate near-optimal values for the following three parameters $(\delta$, $\mu$, and $\sigma^{2}$ ). The final results obtained from five different runs of the GA are shown in Fig. 5 (given the stochastic nature of GAs, the results from different runs of the model typically vary). Specifically, the figure plots the distribution of the optimism index, $\alpha$, using the values obtained for $\mu$ and $\sigma^{2}$, as well as the value obtained for the ambiguity factor, $\delta$. The dashed lines represent the results from the five different runs, whereas the thick solid line represents the average from the five runs. To be noted from Fig. 5 is the observation that the results from the different GA runs are very close to one 


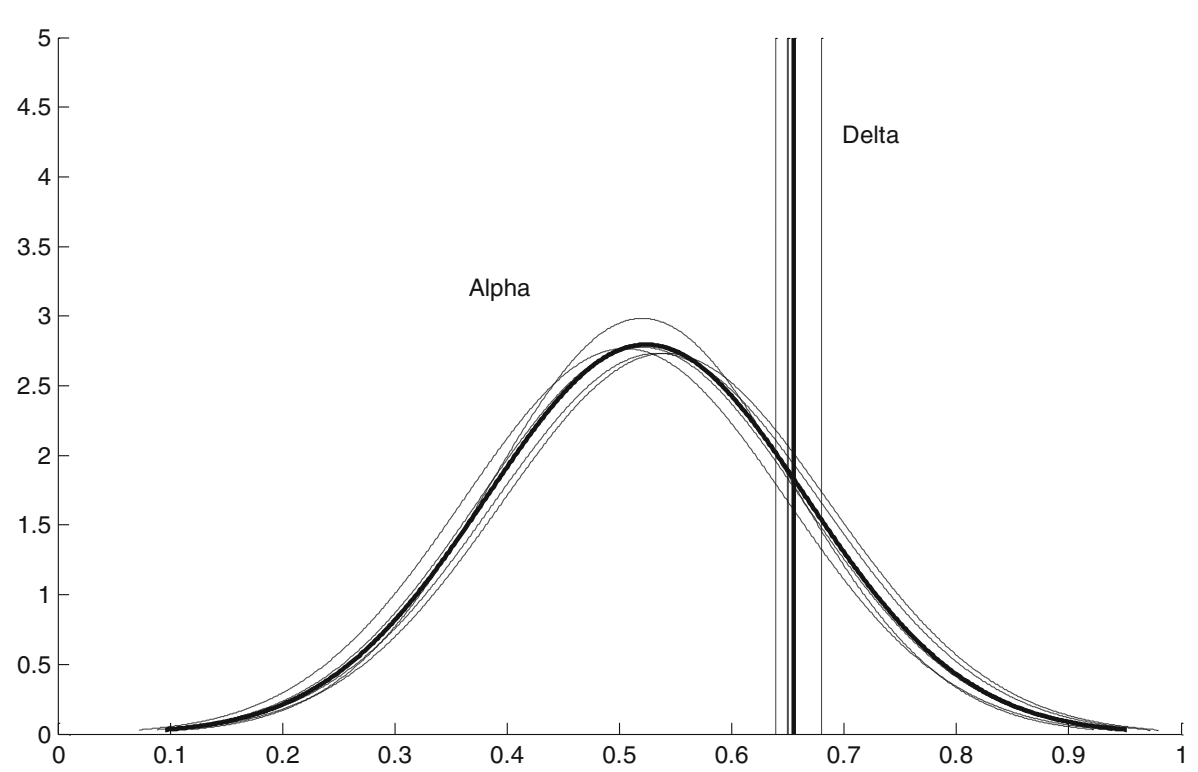

Fig. 5 Calibrated parameters for the dynamic parking model

another, which indicates that the GA appears to be converging on the near "optimal" values for the dynamic parking choice model's parameters.

As can be seen from Fig. 5, for the driver population considered, the index of optimism $(\alpha)$ appears to have a mean value of around 0.576 and a variance of around 0.103 . This means that an average person in that population appears to have a slightly more optimistic attitude toward parking availability in his/her first choice parking on campus. On the other hand, the value of the ambiguity factor $(\delta)$ was found to be around 0.68 , which indicates that the irrational attitude component plays an indispensable part in the parking lot choice decision process. This is obviously attributable to the imperfect information that drivers have regarding parking availability, in the absence of a parking guidance system.

\subsection{Models' predictive accuracy and comparisons}

In order to assess the predictive accuracy of the static and dynamic models, their predictions were compared to the field observations of parking lot choice behavior. Specifically, the number of drivers predicted by the models to go to parking lots $\mathrm{L}_{1}$ and $L_{2}$ were contrasted against the field records values for $\left(\mathrm{N}_{1}+\mathrm{N}_{3}\right)$ and for $\left(\mathrm{N}_{2}+\mathrm{N}_{4}\right)$, respectively. This was done for both datasets 1 and 2, and for the static and dynamic models.

Figure 6 shows the results of the comparisons performed. As can be clearly seen from Fig. 6, the neo-additive capacity dynamic model appears to be providing a more accurate prediction of the numbers of drivers going to $\mathrm{L}_{1}$ and $\mathrm{L}_{2}$ compared to the static model, and thus seems to be more capable of replicating and predicting parking behavior in the parking systems considered. As an example, consider dataset 2 (b) which corresponds to the scenario when the total arrival rate or travel demand is less than the capacity of parking lot $\mathrm{L}_{1}$. For that scenario, it was noticed from field 


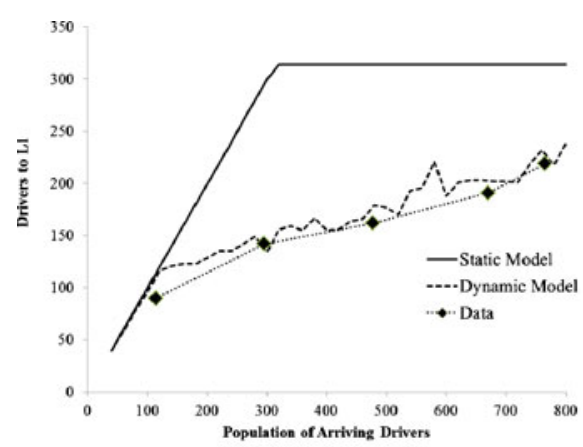

Dataset 1 (a)

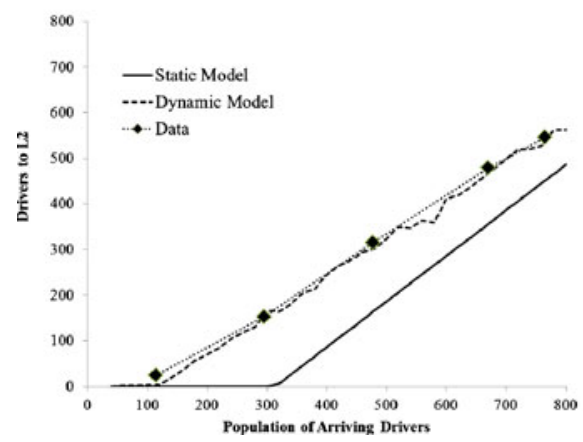

Dataset 1 (b)

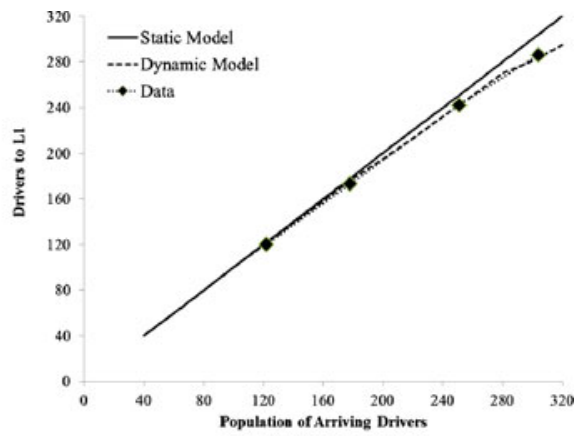

Dataset 2 (a)

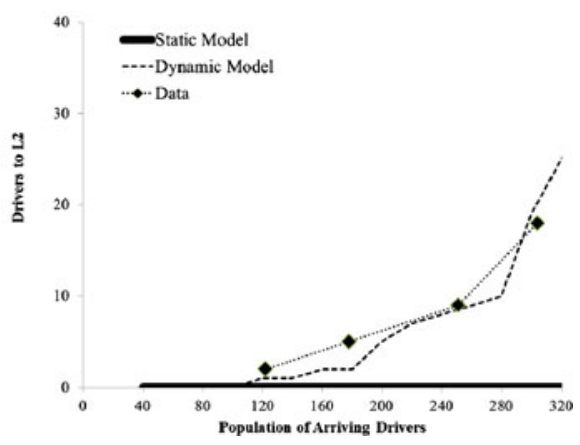

Dataset 2 (b)

Fig. 6 Validation of the two models' predictions of drivers' parking choices

observations that some drivers, in order to avoid the perceived risk of not being able to find an available spot there, decided to go straight to parking lot $\mathrm{L}_{2}$ and park there. The static model however predicted that no driver would go to $\mathrm{L}_{2}$ as can be seen from Fig. 6. Only the dynamic model was able to replicate the observed behavior of drivers, and predicted that some drivers, with a rather pessimistic attitude toward parking availability in $\mathrm{L}_{1}$, would actually go to $\mathrm{L}_{2}$ as was observed. The validation results thus seem to indicate that the drivers' psychological characteristics towards uncertainty significantly affect their parking behavior, and that the dynamic model is more reliable for parking behavior modeling and forecasting.

Another observation that can be made from Fig. 6 is that the static model appears to be giving reasonably accurate results only when the occupancy of parking lot $\mathrm{L}_{1}$ was well below its capacity (i.e. when there were still several spaces available in that parking lot). Under those circumstances, there is a little risk in going to search for spaces in parking lot $\mathrm{L}_{1}$, and hence the majority of drivers make that decision. This explains why the static model was able to capture the parking behavior under such circumstances, because the psychological characteristics of drivers do not play a big role when there is little risk.

\subsection{Scenario analyses}

A key objective of effective parking management is to reduce the magnitude of the additional traffic resulting from the search process (i.e. drivers who go first to their most 
desirable parking lot, only to find it full and hence need to go search for a spot in another parking lot). Using the notation introduced in this paper, that additional volume is equal to $\left(\lambda_{1}-C_{1}-\mu_{1}\right)$, where all notation is as introduced before (we will refer to that volume on the subsequent plots using the term "extra" or additional parking search traffic). Following the development and validation of the models, therefore, the study proceeded to assess the impact of a number of hypothetical scenarios on the additional parking search traffic. Specifically, the study considered the impact of the following three scenarios on the additional parking search traffic volume between the two parking lots in the first dataset: (1) changes in the total parking demand, $\Lambda$; (2) changes in the capacity of the first choice parking lot, $\mathrm{C}_{1}$; and (3) changes in the time cost ratio $\left(\frac{t_{2}+t_{3}}{t_{3}}\right)$. Note that since increasing the departure rate of first choice lot $\mu_{1}$ has the same impact as increasing the first lot's capacity $\mathrm{C}_{1}$, the study did not consider scenarios involving a change in the number of departing vehicles, $\mu_{1}$.

The baseline values for the test scenario considered and for the full observation period lasting from 7:00 to 9:30 am (see Table 5 above) can be summarized as follows: (1) a total demand, $\Lambda=\mathrm{O}\left(\mathrm{L}_{1}\right)+\mathrm{N}_{1}+\mathrm{N}_{2}+\mathrm{N}_{3}+\mathrm{N}_{4}$, equal to 765 vehicles; (2) a total of 25 vehicles departing, $\mu_{1}$; (3) a parking lot capacity for the first choice lot, $\mathrm{C}_{1}$, of 113 vehicles; (4) values for $\mathrm{t}_{1}, \mathrm{t}_{2}, \mathrm{t}_{3}$ equal to 36,110 , and $58 \mathrm{~s}$ respectively; and (5) an observed value for the additional or extra traffic $\left(\mathrm{N}_{3}\right)$ equal to 106 vehicles (i.e. vehicles that went first $\mathrm{L}_{1}$, but failed to find a parking spot there and had to go back to $\mathrm{L}_{2}$ ). For that scenario, the static model predicted a value for $X_{1}{ }^{*}=0.410$, which corresponds to a value for the additional parking search traffic equal to $X_{1}^{*} \Lambda->$ $C_{1}-\mu_{1}=176$. The calibrated dynamic model, on the other hand, predicted a value of 106 , which is identical to the observed value (see Fig. 6).

\subsubsection{Impact of change in the total arrival rate on additional parking search traffic volume}

Figure 7 shows the impact of changes in the total arrival rate of drivers $\Lambda$ on the magnitude of the additional parking search traffic volume; results are shown for both the

Fig. 7 Extra traffic vs. arrival rate

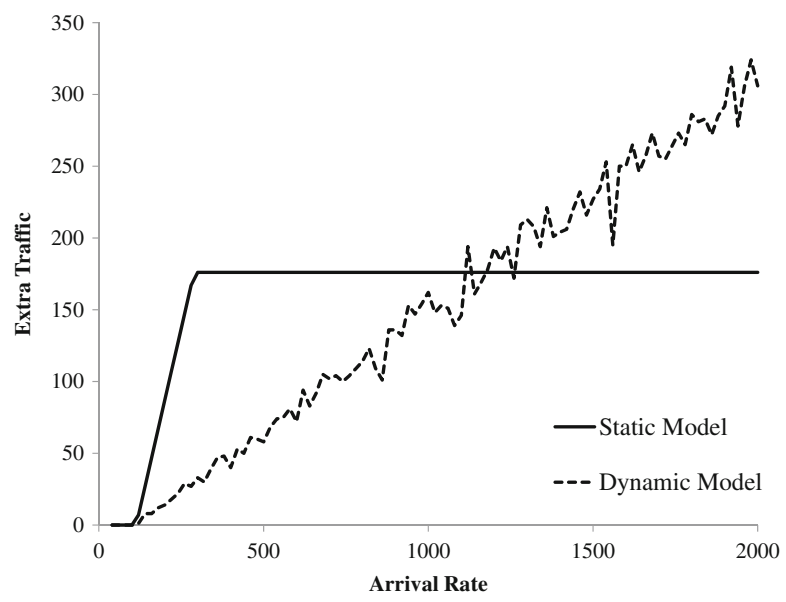


static and the dynamic neo-additive capacity model. Note that the traffic volume plotted on the vertical axis here is different from the number of "Drivers to $\mathrm{L}_{1}$ " previously plotted on Fig. 6, since a subset of the drivers to $L_{1}$ actually succeed in finding a spot in $\mathrm{L}_{1}$. As can be seen, two distinct phases can be distinguished for the static model predictions. In the first phase corresponding to relatively low arrival rates, $\Lambda$ is either less than $\left(\mu_{1}+C_{1}\right)$ or less than $\frac{\left(t_{2}+t_{3}-t_{1}\right)\left(\mu_{1}+C_{1}\right)}{t_{3}}$, and therefore under both those conditions, $X_{1}{ }^{*}=1.0$ (as previously discussed in relation to Table 2 above). Specifically, when $\Lambda$ is between 0 and 138 vehicles, $\Lambda<\left(C_{1}+\mu_{1}\right)$ and therefore there is enough capacity in $\mathrm{L}_{1}$ to accommodate all drivers, and as result the static model predicts 0 additional parking search traffic. For values of $\Lambda$ between 139 and 314 (i.e. the value of $\left.\frac{\left(t_{2}+t_{3}-t_{1}\right)\left(\mu_{1}+C_{1}\right)}{t_{3}}\right)$, the equilibrium condition corresponds to the second case previously outlined in Table 2, which as discussed mentioned in the footnote to Table 2, refers to the case where the probability of failing to find an available spot in $\mathrm{L}_{1}$ is small enough that it is still more advantageous from an expected travel time standpoint to search $\mathrm{L}_{1}$ first. As a result, $X_{1}^{*}$ is also equal to 1.0 , and hence $\lambda_{1}=\Lambda$, in that case. For that interval, the volume of searching traffic would be equal to $(\Lambda-138)$. Finally when the arrival rate exceeds 314 , the parking system satisfies the third condition of reaching equilibrium in Table 2, the parking system is at equilibrium, and the additional search traffic is a constant, since $\lambda_{1}=X_{1}^{*} \Lambda=\frac{\left(t_{2}+t_{3}-t_{1}\right)\left(\mu_{1}+C_{1}\right)}{t_{3}}$ and thus $\lambda_{1}-C_{1}-\mu_{1}=176$ vehicles. The fact that the additional search traffic remains constant for all values of $\Lambda$ above 314 vehicles, points to a limitation of the static model which stems from the fact that the model ignores the psychological characteristics of drivers.

On the other hand, the predictions of the dynamic or sequential, neo-additive capacity model appear to agree more with intuition. As can be seen from Fig. 7, the dynamic model predicts an increase in the amount of additional or parking search traffic with increases in the total traffic demand desiring to park. The trend appears to be linear (please note that the oscillations in the figure are a result of the stochastic elements in the model in terms of the distribution of the optimistic and pessimistic attitudes of the driver population, for example). The dynamic model predictions are also much closer to the observed base case value, as previously discussed. Another observation that could also be made is that, for values of $\Lambda$ less than around 1200 vehicles, the irrational aspect of decision-making, which reflects the psychological characteristics of drivers in terms of their optimistic and pessimistic attitudes, appear to benefit the parking system, since the amount of traffic predicted by the dynamic model is less than that predicted by the static model which only accounts for the rational aspect of decision-making.

\subsubsection{Impact of change in the capacity of the first choice parking lot on additional parking search traffic volume}

Figure 8 shows the impact of changes in the capacity of the first choice parking lot on the amount of additional parking search traffic volume generated, as predicted by both the static and dynamic models. Once again, the predictions of the static model appear to significantly differ from those of the dynamic model. For values of $\mathrm{C}_{1}$ satisfying the condition $\Lambda \geq \frac{\left(t_{2}+t_{3}-t_{1}\right)\left(\mu_{1}+C_{1}\right)}{t_{3}}$, the equilibrium value $X_{1}{ }^{*}=\frac{\left(t_{2}+t_{3}-t_{1}\right)\left(\mu_{1}+C_{1}\right)}{t_{3} \Lambda}$, which means that the additional traffic can be calculated as follows: $\lambda_{1}-C_{1}-\mu_{1}=\Lambda$. 
Fig. 8 Extra traffic vs. capacity change

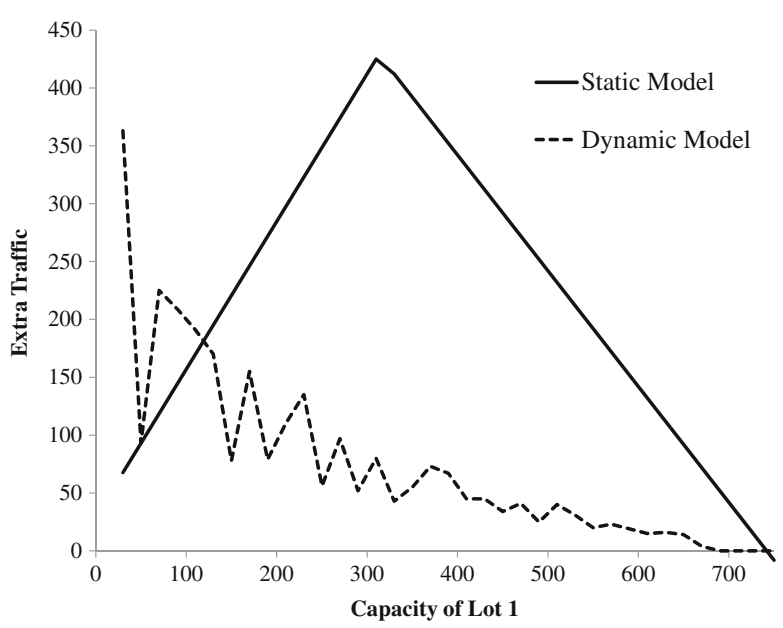

$\frac{\left(t_{2}+t_{3}-t_{1}\right)\left(\mu_{1}+C_{1}\right)}{t_{3} \Lambda}-C_{1}-\mu_{1}=\frac{\left(t_{2}-t_{1}\right)\left(\mu_{1}+C_{1}\right)}{t_{3}}$. Given this, for that range of values (specifically for $\mathrm{C}_{1}$ between 0 and 313 ), the magnitude of the additional parking search traffic is predicted to increase as the capacity of the lot increases. According to the static model, therefore, the implication is that investments in increasing the size of the first choice parking lot would lead to the undesirable result of generating additional search traffic, since there is now a higher probability of finding an available parking space there, which would in turn encourage more drivers to search that lot first.

Further increases in the capacity of the first choice parking plot would lead to a value of $\Lambda$. When this happens, $X_{1}{ }^{*}$ becomes equal to 1.0 , and accordingly, the value of the additional parking search volume can be calculated as $\lambda_{1}-C_{1}-\mu_{1}=\Lambda->C_{1}-\mu_{1}$ , which means that in that case, the additional traffic volume will decrease as the capacity increases. This continues until a value for the capacity equal to 740 spaces, when the magnitude of the additional parking search traffic drops to 0 , because the total demand is less than the capacity of the lot minus the number of vehicles departing $(765-25=740)$.

Considering now the dynamic or sequential model predictions, one immediately notices a generally decreasing trend in the magnitude of the additional parking search traffic with increases in the capacity of the first choice parking lot (once again the oscillations are due to the stochastic elements in the dynamic model). Moreover, the rate of decrease in the magnitude of the traffic volume appears to slow down with increases in the capacity. For example, it seems that having a parking lot with a capacity of less than 100 spaces would result in a dramatic increase in the parking search traffic. For values above 100 spaces, the additional traffic volume decreases, but at a much lower rate than its rate of decrease for capacity values less than 100 spaces. Analyses such as these may therefore be beneficial in determining the minimum size of the most desirable parking lot.

\subsubsection{Impact of change in the time cost structure on additional parking search traffic volume}

The study also investigated the impact of changing the relative values of the different time cost elements associated with the problem formulation. As previously 
mentioned, three different variables were defined to capture the different time cost elements: $t_{1}$, which captures the time cost involved in going to $L_{1}$ and successfully parking there; $t_{2}$, which captures the time cost of going to $L_{2}$ right away, parking there and walking a longer distance to the destination building; and $t_{3}$, which captures the additional time incurred for a driver who goes first to $L_{1}$, finds the lot full and thus drives back to $L_{2}$. A time cost ratio was thus defined as $r=\left(t_{2}+t_{3}\right) / t_{2}$, to represent the relative magnitude of the "penalty of risk seeking" (i.e. seeking to find a spot first in $\mathrm{L}_{1}$ ) over the "cost of risk avoidance" (i.e. going straight to $\mathrm{L}_{2}$ ).

Figure 9 shows the likely change in the magnitude of the additional search traffic volume, predicted by both the static and dynamic models, as the value of $\mathrm{r}$ changes from 1.25 to 1.70 . As can be seen, while both the static and dynamic model appear to predict a decrease in the magnitude of the search traffic volume with increasing values of the ratio, $r$, there are still noticeable differences between the two models' predictions especially at higher values of the ratio. Specifically, for ratios less than around 1.45, the predictions of the two models appear to be close to one another. On the other hand, starting from values of $\mathrm{r}$ greater than 1.50, there is a noticeable divergence in the predictions of the two models. For example at a value of $\mathrm{r}$ equal to 1.70 , the dynamic model predicts zero additional traffic, whereas the static model still predicts close to around 100 vehicles who would go first to $L_{1}$, find it full and drive back to $L_{2}$. Intuitively, one would expect that as the value of the penalty of risk seeking increases relative to the cost of risk avoidance, a majority of drivers would prefer to go to the second parking lot right away. The dynamic model, because it accounts for the psychological characteristics of drivers, appears to be capable of reflecting that phenomenon, whereas the static model could not.

\section{Conclusions and future research}

\subsection{Research conclusions}

In this paper, two types of parking choice models utilizing game theory were developed. Because the static model pays no attention to modeling individual drivers'

Fig. 9 Extra traffic vs. time cost ratio

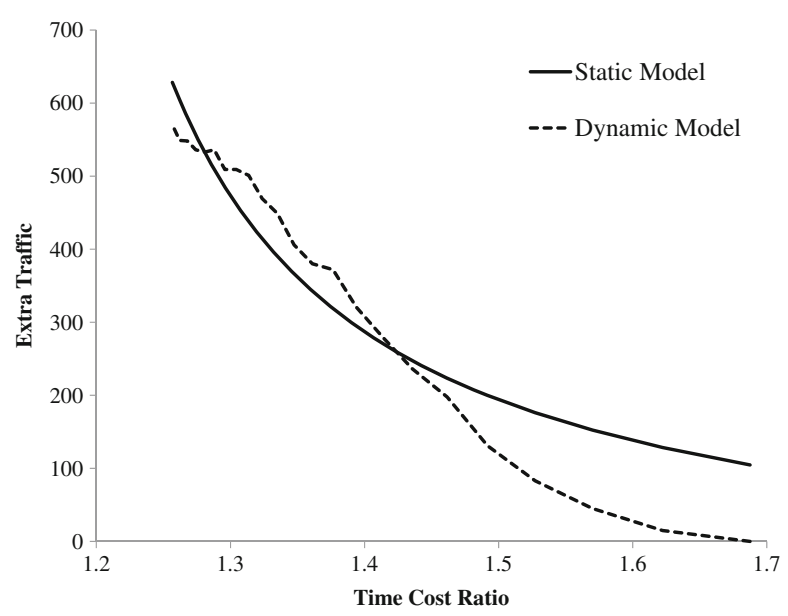


psychological characteristics, it represents an aggregate model designed to only predict the percentage of drivers that go to a given parking lot, rather than predicting the individual choice of a certain driver with certain psychological characteristics. The dynamic model, on the other hand, includes a custom-defined neo-additive capacity formulation which allows it to capture differences among individual drivers' psychological characteristics in terms of their optimistic and pessimistic attitudes toward a parking system under uncertainty. The dynamic or sequential model is therefore a disaggregate behaviorally-based model, fit for incorporation within an activity-based or agent-based transportation modeling framework. Among the main conclusions derived from the study are:

(1) The dynamic or sequential neo-additive capacity model appears to be quite capable of accurately replicating and predicting drivers' observed parking choice behavior. This was quite evident from the results shown on Fig. 6, where the dynamic model predictions were quite close to field observations.

(2) Compared to the static game-theoretic model, the sequential neo-additive capacity model appears to offer superior predictive accuracy, as was also shown on Fig. 6. Moreover, the results of the sensitivity analysis conducted on the two models demonstrate that the insights gained from the dynamic model are more intuitive and logical compared to the static model. This in turn seems to indicate that drivers' psychological characteristics towards uncertainty, and their pessimistic or optimistic attitudes regarding parking availability, play a significant role in the parking choice decision-making process and hence need to be rightly accounted for in modeling.

(3) For the same driver population, the results from the study indicate that the parameters of the sequential neo-additive capacity model (namely the mean and standard deviation for the optimistic index, $\alpha$, and the ambiguity factor, $\delta$ ) are transferable from one parking system to another. This was evident from the fact that the calibrated model for the first pair of parking lots (dataset 1) yielded very high predictive accuracy when applied to the second pair of lots (dataset 2).

(4) For the driver population considered in this study (faculty, staff and students at UB), drivers, on average, seemed to have a slightly more optimistic view regarding parking availability in their most desirable parking lot (i.e. the optimistic index $(\alpha)$ had a mean value of 0.576$)$. Moreover, the irrational side of decision making (indicated by a value for the ambiguity factor $\delta$ close to 0.7 ) appeared to constitute an indispensable component of their parking lot choice decision process.

(5) Given conclusion (2) and (4) above, which clearly demonstrate the significant role that drivers' optimistic and pessimistic attitudes play in their parking lot choice decision-making process, an effective parking management and information system has the potential to significantly reduce the magnitude of the additional traffic volume resulting from the parking search process.

\subsection{Limitations and future research}

There are several future research directions which the authors are planning to pursue in order to complement the current work. Three such directions are listed below: 
(1) The parking systems considered in this study consisted of a smaller, more desirable parking lot as opposed to a much larger, less desirable lot, which in fact represented the union of all remaining alternative lots as previously described. In their future research, the authors plan to test the model on parking systems consisting of more than two lots. For that case, the authors will employ a hierarchical decision-making process, whereby decisions would be made in a sequential fashion, as previously described. This means that, following a driver's choice of his/her most desirable lot, $\mathrm{L}_{1}$, the alternative "aggregate" lot, $\mathrm{L}_{2}$, would then split into a desired lot (i.e. the second choice parking lot) and an alternative lot (represents the union of all remaining lots), as previously explained in Section 2.1.

(2) Given that the model described in this work was developed and tested on a university campus parking system, future research will focus on applying the model to other parking environments that are significantly different from the case study herein.

(3) Finally, the authors plan to incorporate the dynamic, neo-additive capacity parking choice model within an activity-based transportation model which the authors recently developed for the UB north campus. This will allow for accurate modeling of parking behavior and will yield a method for accurately quantifying the additional environmental cost of the parking search process, in terms of wasted fuel consumption and increased emissions.

\section{References}

Altman E, Wynter L (2004) Equilibrium, games, and pricing in transportation and telecommunication networks. Network Spatial Econ 4(1):7-21

Cassady CR, Kobza JE (1998) A probabilistic approach to evaluate strategies for selecting a parking space. Transp Sci 32(1):30-42

Chalamish M, Sarne D, Kraus S (2007) Mass programmed agents for simulating human strategies in large scale systems. Paper presented at the Proceedings of the 6th international joint conference on Autonomous agents and multiagent systems, Honolulu, HI

Chateauneuf A, Eichberger J, Grant S (2007) Choice under uncertainty with the best and worst in mind: Neo-additive capacities. J Econ Theor 137(1):538-567

De Palma A, Ben-Akiva M, Brownstone D, Holt C, Magnac T, McFadden D et al (2008) Risk, uncertainty and discrete choice models. Mark Lett 19(3):269-285

Ellis RH, Rassam PR (1970) Structuring a systems analysis of parking. Highway Res Rec (317), 1-13.

Florian M, Los M (1980) Impact of the supply of parking spaces on parking lot choice. Transportation Research Part B: Methodological 14(1-2):155-163

Goyal SK, Gomes LFAM (1984) A model for allocating car parking spaces in universities. Transportation Research Part B: Methodological 18(3):267-269

Gur YJ, Beimborn EA (1984) Analysis of parking in urban centres: equilibrium assignment approach. Transp Res Rec 957:55-62

Hunt JD (1988) Parking location choice: insights and representations based on observed behaviour and hierarchical logit modelling formulation. Institute of Transportation Engineers, 58th Annual Meeting, Compendium of Technical Papers, Vancouver, Canada, 439-446

Khattak A, Polak J (1993) Effect of parking information on travelers' knowledge and behavior. Transportation 20(4):373-393

Leephakpreeda T (2007) Car-parking guidance with fuzzy knowledge-based decision making. Build Environ 42(2):803-809

Lin C-C, Hsieh C-C (2012) A cooperative coalitional game in duopolistic supply-chain competition. Network Spatial Econ 12(1):129-146 
Michalewicz Z (1994) Genetic algorithms + data structures = evolutionary programs. Springer-Verlag Press Neema MN, Maniruzzaman KM, Ohgai A (2011) New genetic algorithms based approaches to continuous p-median problem. Network Spatial Econ 11(1):83-99

Nour Eldin MS, El-Reedy TY, Ismail HK (1981) A combined parking and traffic assignment model. Traffic Eng Contr 22(10):524-530

Polak J, Axhausen K (1990) Parking search behaviour: a review of current research and future prospects: Oxford University.

Sarin R, Wakker P (1998) Revealed likelihood and knightian uncertainty. J Risk Uncertainty 16(3):223250

Schmeidler D (1989) Subjective probability and expected utility without additivity. Econometrica 57 (3):571-587

Shepherd S, Sumalee A (2004) A genetic algorithm based approach to optimal toll level and location problems. Network Spatial Econ 4(2):161-179

Thompson RG, Richardson AJ (1998) A parking search model. Transportation Research Part A: Policy and Practice 32(3):159-170

TsukaguchiH, Jung H (1989) Basic considerations on parking strategy in central business districts to obtain a better use of the limited available parking space. Proceedings of the 5th World Conference on Transportation Research, Yokohama, Japan, 309-322

Tversky A, Kahneman D (1992) Advances in prospect theory: cumulative representation of uncertainty. J Risk Uncertainty 5(4):297-323

Van Der Goot D (1982) A model to describe the choice of parking places. Transport Res Gen 16(2):109115

Von Neumann J, Morgenstern O (1944) Theory of games and economic behavior: Princeton University Press.

Wakker PP (2001) Testing and characterizing properties of nonadditive measures through violations of the sure-thing principle. Econometrica 69(4):1039-1059

Wang X, Zhuang J (2011) Balancing congestion and security in the presence of strategic applicants with private information. Eur J Oper Res 212(1):100-111

Young W (1986) PARKSIM/1: a network model for parking facility design (Vol. 27). London, ROYAUME-UNI: Hemming

Young W, Weng TY (2005) Data and parking simulation models. In Simulation approaches in transportation analysis. Operations Research/Computer Science Interfaces Series, Volume 31, Part III, pp. 235267

Young W, Taylor MAP, Thompson RG, Ker I, Foster J (1991) CENCIMM: a software package for the evaluation of parking systems in central city areas. Traffic Eng Contr 32(4):186-193

Zhang P, Peeta S, Friesz T (2005) Dynamic game theoretic model of multi-layer infrastructure networks. Network Spatial Econ 5(2):147-178 